\title{
LA PERSPECTIVA DE UN TERAPEUTA GESTÁLTICO SOBRE LA INDAGACIÓN TERAPÉUTICA
}

\section{A GESTALT THERAPIST'S PERSPECTIVE ON THERAPEUTIC INQUIRY}

\author{
Fermín González Cuellar \\ Psicólogo especialista en psicología Clínica ANO-02475, en \\ Centro Sanitario Escuela Sevilla de Terapia Gestalt Jera en Sevilla. España \\ ORCID: https://orcid.org/0000-0002-0809-2398
}

Cómo referenciar este artículo/How to reference this article:

González-Cuellar, F. (2021). La perspectiva de un terapeuta gestáltico sobre la indagación terapéutica. Revista de Psicoterapia, 32(118), 47-58. https://doi.org/10.33898/rdp.v32i118.484

\begin{abstract}
Resumen
Es de vital importancia que el terapeuta en el proceso de indagación de la historia y la experiencia fenomenológica del cliente muestre interés, respeto, implicación y disponibilidad por y para él/ella. Por ende, el terapeuta ha de evitar las interpretaciones, los juicios y los prejuicios y las confrontaciones críticas sobre las respuestas, formas de ser y estar de la persona. Es un proceso para conocer la historia y a la vez sintonizar con el ritmo, los afectos, los aspectos evolutivos y las necesidades relacionales de la persona. De este modo, se crea un vínculo dotado de seguridad y validación que permite descubrir cómo la persona cimienta el contacto consigo y con los demás. En síntesis, esta actitud llevada con conciencia continua por parte del terapeuta es relevante en el proceso de indagación para que este se convierta en una puerta de entrada a la reparación de daños psicológicos.

Palabras claves: actitud, indagación, terapia gestalt, psicoterapia integrativa, respeto
\end{abstract}

\begin{abstract}
It is of utmost importance that the therapist shows interest, respect, involvement, and availability by and for the client, during the process of inquiry into his/her history and phenomenological experience. Therefore, the therapist has to avoid interpretations, judgments and prejudices, and critical confrontations regarding the person's responses and ways of being. It is a process to get to know the story and at the same time to attune to the client's rhythm, affects, developmental aspects, and relational needs. In this way, a bond endowed with security and validation that allows discovering how the client establishes contact with himself/herself and with others is created. In summary, this attitude taken with an ongoing awareness by the therapist is relevant in the inquiry process so that it becomes a gateway to repair psychological damage.
\end{abstract}

Keywords: attitude, inquiry, gestalt therapy, integrative psychotherapy, respect 
En Terapia Gestalt: excitación y crecimiento de la personalidad humana los autores describen la vital importancia de la actitud del psicoterapeuta a partir de la cual indaga la historia y la experiencia fenomenológica del cliente (Perls et al., 1951; Rosenfeld, 1978). En el libro Más allá de la empatía: una terapia de contacto en la relación, los autores afirman que la indagación terapéutica implica interés, respeto, disponibilidad, apertura para escuchar la historia y la experiencia del cliente sin dar nada por sentado y dejarse impactar (Erskine et al., 1999/2012). Tanto la Terapia Gestalt como la Psicoterapia Integrativa, hacen hincapié en la importancia de la indagación terapéutica (Perls y Baumgardner, 1975; Moursund y Erskine, 2003)

Erskine y sus coautores aplican esta perspectiva filosófica cuando afirman que es necesario evitar interpretaciones, juicios y prejuicios sobre las maneras de comportarse del cliente, sus actitudes y el estilo de contacto interpersonal que aportan a la relación terapéutica. La indagación efectiva incluye la sincronía del terapeuta con su ritmo, la sintonía con su afecto, la comprensión de cómo se desarrollaron y el interés en cómo se relacionan con las personas en su vida (Erskine, 2015). Perls (1969) utilizó la indagación para desafiar las formas fijas de pensar y comportarse de los clientes.

La indagación terapéutica es una puerta de entrada para reparar el daño psicológico de nuestros clientes, daño que puede ser emocional, físico, o en cómo se valoran a sí mismos. El terapeuta se convierte en una persona importante en el proceso terapéutico porque la actitud que mantiene durante su comunicación interpersonal tiene una influencia significativa en el resultado de la psicoterapia del cliente (Erskine, 2013).

El verbo "inquirir" proviene del latín y se utiliza para nombrar la intención de saber algo a través de la investigación o de las preguntas (Pérez Porto y Gardey, 2019). En el contexto terapéutico, la indagación trata de ir más allá de conocer algo; trata de conocer a alguien de una manera interpersonal profunda, una búsqueda de lo que le sucede internamente así de cómo lo vivencia y lo expresa. La indagación terapéutica implica aprender sobre la otra persona, llegar a conocer realmente su narrativa personal, tanto explícita como implícitamente. Es una forma de hacer preguntas basadas en un interés genuino en la otra persona, un interés libre de prejuicios, suposiciones y críticas.

En relación a las aportaciones de Perls (1944, 1969, 1973), se describen los objetivos de la Terapia Gestalt que ilustra cómo la indagación terapéutica ayuda a enfocar la conciencia del cliente en el proceso de descubrirse a sí mismo mientras que, al mismo tiempo, el terapeuta trata de conocer la historia de vida y la situación actual del cliente. Cuando se utiliza la indagación terapéutica la intención es sentir al otro, conocerlo plenamente sin avergonzarlo o retraumatizarlo y, además, explorar los procesos cognitivos, emocionales, corporales y conductuales del cliente y llevarlos plenamente a su conciencia.

Erskine (2021), describe cómo la indagación es una vía de entrada a la participación interpersonal y a la sintonía con el otro. No sólo estimula al cliente, sino 
que también desarrolla la creatividad, la espontaneidad y la sana curiosidad del terapeuta para conocer y comprender al otro. La indagación terapéutica es un arte de sutileza y cuidado del terapeuta hacia el cliente desarrollando una comunicación terapéutica efectiva. Es nuestra tarea profesional entender el aprendizaje de las perspectivas únicas de los clientes, para sentir sus emociones, y responder a las dificultades del cliente a medida que se van presentando en la relación terapéutica.

Yontef (1993/1995), un muy respetado terapeuta gestáltico, señala que la indagación es "un arte basado en el darse cuenta fenomenológico, en el contacto dialogal dentro del modelo relacional Yo-Tú, donde no hay presión para que renuncien a su realidad" (p. 221). Para muchas personas es una experiencia nueva y única y cuando se respetan sus respuestas, sin dudar o cuestionarlas, el proceso de indagación refleja un interés auténtico, libre de prejuicios, validando el proceso de introspección de nuestro cliente. En resumen, la indagación terapéutica implica una apertura a conocer a las personas por lo que son y por la historia personal que aportan a nuestras sesiones de terapia; implica también, ayudarles a descubrir las funciones psicológicas de sus sensaciones, sentimientos, pensamientos y comportamientos corporales (Erskine, 2015; Yontef, 1993/1995; Zinker, 1978).

\section{Finalidades de la Indagación Terapéutica}

Conocer y comprender plenamente a nuestros clientes lleva un tiempo considerable. Hay que crear un clima de respeto y confianza para que ellos tomen conciencia de quiénes son y cómo se manejan y eventualmente asumir la responsabilidad de lo que él o ella piensa y cómo actúa (Fagan y Shepherd, 1970; Perls, 1969). Además, la indagación del terapeuta debe ser:

Empática con la experiencia subjetiva del cliente para ser eficaz en el descubrimiento y la revelación de los fenómenos internos (sensaciones físicas, sentimientos, pensamientos, significados, creencias, decisiones, esperanzas y recuerdos) y en el descubrimiento de las interrupciones internas y externas del contacto (Erskine et al., 1999/2012, p. 56).

Estas mismas ideas se repiten en el escrito de Hycner y Jacobs (1995) sobre las condiciones necesarias para crear una relación curativa en la Terapia Gestalt.

Es esencial que la indagación atienda a las necesidades relacionales de los clientes y si sus diversas necesidades han recibido respuestas adecuadas o no (Erskine, 2004). Hay que enfocar tanto las necesidades del aquí y ahora, como las necesidades relacionales que no fueron satisfechas debido a la negligencia acumulada o a los traumas en la infancia del cliente. Yontef (1993/1995) lo dice de esta manera: "la indagación tiene que dirigirse hacia el cliente que la experimenta como un proceso de atención a sus necesidades relacionales, a la necesidad dominante; la conciencia (si la persona no sabe lo que necesita, la conciencia pierde significado)" (p. 171).

La relación terapéutica en sí misma puede generar conciencia de los procesos internos en los que surgen las necesidades relacionales, y se expresan a través del proceso de diálogo Yo-Tú (Buber, 1958). 
La indagación terapéutica ayuda a revelar la historia inconsciente y los conflictos de desarrollo de los clientes. En la figura 1, se resume cómo visualizo las diversas áreas de indagación: actuación, afecto, fisiología, contenido y estilo narrativo, sueños y necesidades.

Figura 1. Aspectos de la indagación en el cliente. (Adaptado de Erskine, 2015)

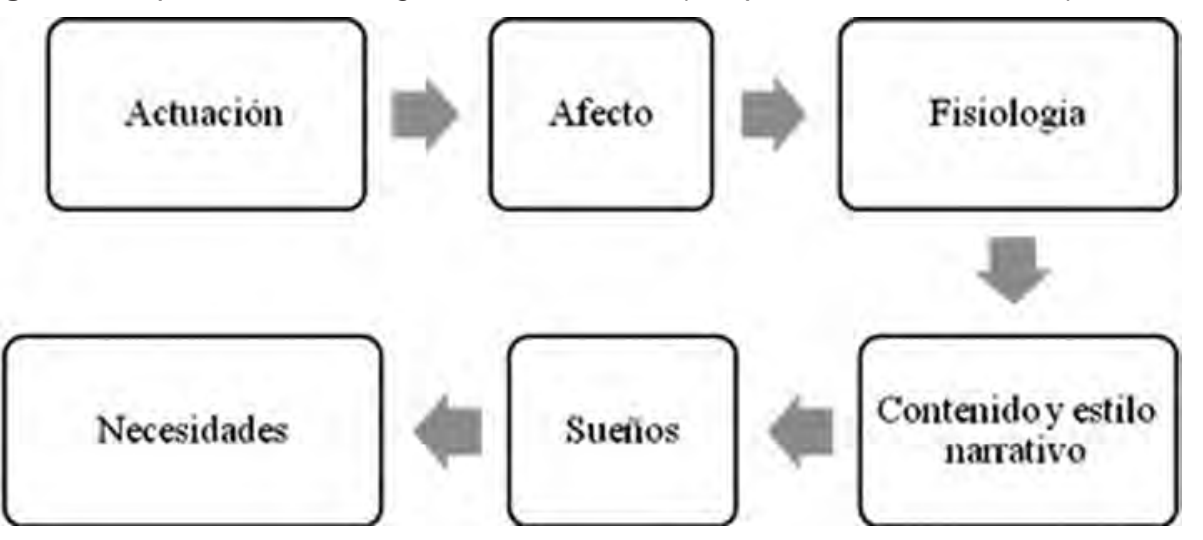

Tanto el contenido como el estilo del proceso de respuesta de los clientes revela los conflictos emocionales que hay en su vida, tales como: abandono emocional, abuso, miedo, preocupaciones, ira, desesperación y tensiones corporales. En el proceso se enfoca continuamente las experiencias sensoriales y motoras de los clientes, como la forma en que respiran, tensan sus músculos y mantienen su postura. Se presta mucha atención a los sueños y fantasías del cliente porque a menudo revelan cómo el cliente se define a sí mismo, lo que anticipa de los demás y cómo se imagina su calidad de vida. Y se presta atención a la forma en que el cliente mantiene o interrumpe el contacto tanto interno como externo (Perls et al., 1951).

Se abre un camino para que la persona descubra los aspectos ocultos de sí mismo, aspectos importantes de sí mismo que no han sido desarrollados. A través de una indagación respetuosa que incluye el reconocimiento, la validación y la normalización, los clientes son cada vez más capaces de descubrirse a sí mismos.

En resumen, la indagación terapéutica efectiva se basa en la capacidad del terapeuta de expresar respeto, honestidad, sintonía, validación e implicación: estos son algunos de los factores centrales que crean una relación de curación (Erskine, 2021).

\section{Características de la Indagación}

La indagación terapéutica tiene varios niveles: fenomenológico, histórico, estrategias de adaptación y el valor del yo. En los comienzos de la psicoterapia con un cliente es conveniente entretejer la indagación fenomenológica con la histórica mientras se observa las interrupciones del cliente en el contacto interno o externo, 
que revelan las estrategias adaptativas del cliente y la historia inconsciente que se revela en la transferencia con el psicoterapeuta.

Yontef (1993/1995) enfatiza que cada cliente necesita ser escuchado, reconocido y que su experiencia fenomenológica sea respetada. Esto requiere que el psicoterapeuta confíe y respete las experiencias emergentes y las necesidades relacionales de los clientes. Erskine está de acuerdo con la perspectiva de Yontef que define la indagación fenomenológica como una "indagación del aquí y ahora de la experiencia interna del cliente" (2015, p. 29). Esta pues, requiere que uno se sintonice con los ritmos de los clientes y que permanezca sensible a ellos.

La indagación fenomenológica incluye hacer preguntas a los clientes sobre las siguientes áreas de experiencia interna:

a) Sensaciones físicas y reacciones corporales.

b) Afectiva: conciencia de los afectos y como el cliente los expresa.

c) Cognitivo: recuerdos explícitos, conclusiones, decisiones, significados, expectativas, esperanzas, fantasías y deseos.

d) Necesidades: satisfacción o no satisfacción de las necesidades relacionales.

e) Comportamiento: comportamientos aceptados o comportamientos evitados.

f) Relacional: la calidad del contacto interpersonal; la capacidad de ser auténtica y plenamente presente. (Adaptado de Erskine et al., 1999/2012, pp. 76-84)

La indagación histórica es una investigación de los eventos claves del pasado del cliente que pueden haber formado su personalidad. Incluye un enfoque en las interrupciones o satisfacciones relacionales que pueden haber ocurrido en varias edades. Y a menudo, se transforma en una exploración sobre cómo el niño se las arregló para estabilizar y regular su afecto, de cómo enfrentó los factores estresantes en su vida, las fantasías, deseos y repulsiones de la infancia de los clientes; y las necesidades relacionales que pueden o no haber sido satisfechas.

También incluye preguntas de: quién, qué, cuándo, dónde y por qué. Estas son preguntas de hecho, diferentes a las que se utilizan en la indagación fenomenológica que es sobre la experiencia interna de los clientes, de sus sensaciones corporales, afectos, fantasías, recuerdos y pensamientos. Cuando preguntamos sobre cómo un niño puede haber enfrentado las alteraciones relacionales, a menudo entrelazamos preguntas factuales sobre su historia con otras fenomenológicas sobre sus experiencias subjetivas.

La historia del cliente se revela a menudo tanto en la transferencia como en la contratransferencia del psicoterapeuta. Por ejemplo, un posible deseo de rescate, irritación o aburrimiento, o el deseo de criticar puede ser una reacción contratransferencial a las historias inconscientes de los clientes sobre las alteraciones relacionales en sus vidas. Utilizando sus reacciones internas, el terapeuta puede tomar estas reacciones contratransferenciales y convertirlas en una serie de indagaciones fenomenológicas que ayuden al cliente a descubrir su historia relacional.

La indagación efectiva implica sintonizarnos con el ritmo de los clientes 
(Moursund y Erskine, 2003). Una metáfora que describe la sintonía rítmica necesaria que acompaña a la indagación está en la historia del remolcador y el barco. Un remolcador está unido a un gran barco para guiarlo hasta el puerto y atracar de forma segura. Un atraque seguro requiere que el capitán del remolcador sienta el ritmo del barco, el flujo de las corrientes y los ajustes necesarios en el movimiento a medida que el barco se desliza hacia un lugar seguro. De manera similar, el ritmo del psicoterapeuta, los tonos de voz, la consistencia y la fiabilidad, son aspectos importantes de la indagación efectiva que guía al cliente hacia el autodescubrimiento. El silencio también puede ser parte del ritmo de la indagación al proporcionar el tiempo para la introspección del cliente. Y la mirada apreciativa que refuerza la indagación y el acceso a las partes profundas e íntimas donde se muestra la verdad de la persona, su historia sin censura. Si la indagación es agresiva en términos de tono, palabras, posturas corporales o mirada inquisitiva, el cliente puede reaccionar huyendo, atacando o adormeciéndose.

\section{Recorrido Experiencial}

Este artículo está basado en mi experiencia profesional de muchos años como psicoterapeuta. Representa mi interés en el concepto de indagación terapéutica y en cómo podemos implementar estas ideas en varios escenarios como la psicoterapia individual, la psicoterapia de grupo, o las sesiones de procesos de grandes grupos relacionales. Descubrí que la indagación terapéutica no es sólo sobre mis clientes, sino que también se aplica a mí mismo. Hay momentos en los que debo hacerme preguntas sobre mi contratransferencia. Por ejemplo: “¿Qué está pasando dentro de mí que no me concentro en lo que mi cliente está diciendo?”; “¿Qué quiero que el cliente piense de mí?”; o, “¿Cuáles son mis expectativas y necesidades relacionales?”. Me hago este tipo de preguntas por respeto, interés e implicación con el proceso terapéutico. Me recuerda a Perls diciendo, "la otra persona no está ahí para satisfacer mis expectativas” (citado por Peñarrubia, 1998, p. 165).

Para algunos clientes la indagación puede resultar incómoda, en particular si tienen un historial de haber sido definido por otros o haber sido objeto de burlas, acusaciones o interrogatorios. Vienen a la terapia anticipando que tal desatención relacional vuelva a ocurrir, y responden con vergüenza y pueden censurarse aquello que tienen por decir. Es en momentos como este que necesitamos practicar el arte de la psicoterapia, un arte relacional basado en una filosofía que honra las experiencias afectivas de los clientes, que encuentra sentido en las fantasías y expectativas de los clientes, y una actitud que es la comprensión de los conflictos internos de los clientes en lugar de ver al cliente como patológico (Erskine, 2013).

En algunas ocasiones, los clientes han mostrado síntomas o me han contado historias que me han confundido o perturbado. Mi experiencia reactiva a menudo ha reflejado los intentos de los clientes de distraerse del contacto interno con los sentimientos y recuerdos; son un tipo de “desviación” (Polster y Polster, 1973, p. 89-92), "estrategias de afrontamiento para situaciones conflictivas y generadoras 
de estrés” (Macías et al., 2013, p. 125).

Encuentro que algunos clientes responden a mis preguntas sobre su historia o proceso interno con un inmediato "no sé". Según el cliente, se puede escuchar el "no sé" como si tuviera una variedad de significados: la frase puede transmitir una sensación abrumadora de vergüenza, puede significar que no tienen las palabras para expresar sus sensaciones, las palabras pueden significar que no tienen curiosidad por descubrir su propia experiencia interna, puede significar que no pueden traducir un recuerdo implícito a uno explícito; o estas palabras pueden transmitir una expresión de desesperanza o ira.

Los clientes usan otras palabras que expresan una variedad similar de significados: “Por qué?”; “¿Para qué?”; o "es inútil”. He encontrado que estas frases son, a menudo, una expresión inconsciente de una creencia fundamental “Algo está mal en mí”, la creencia que es central para la experiencia de la vergüenza (Erskine, 1995). Estas frases interrumpen el contacto interpersonal, pero su propósito es mitigar la incomodidad interna, para evitar el dolor y la ira. Estas interrupciones del contacto interno proporcionan un alivio temporal; reducen la conciencia y la angustia del trauma o el abandono acumulado.

Perls (1969) describió la importancia de indagar cómo se recrean repetidamente viejos recuerdos de experiencias humillantes para mantener la homeostasis con las creencias de guión y la negación de las necesidades y sentimientos originales, tal y como recoge Erskine, (2015). Para ello el niño olvida, se desconecta de su sensación interna y busca ajustarse a las necesidades de su cuidador. Esta acomodación genera conclusiones basadas en experiencias que servirán para distraer a la persona de la memoria implícita de soledad, vacío y desajuste que puede haber experimentado.

Van der Kolk (2014/2015) describe su propio descubrimiento de lo doloroso que puede ser enfrentarse a la realidad del trauma relacional. En el mismo trabajo cuenta como su gran maestro Elvin Semrad enseñaba que "la mayor parte de nuestro propio sufrimiento son las mentiras que nos decimos a nosotros mismos" (citado en Van der Kolk, 2014/2015, p. 28). Incluso con una indagación respetuosa, los clientes tienden inconscientemente a engañarse a sí mismos, temen sentir el dolor y evitan hablar de su experiencia real. En consecuencia, hay muchas personas que, sin darse cuenta, se mienten a sí mismas, se cuentan a sí mismas un mito y luego le cuentan esa misma historia al terapeuta. Si el terapeuta desaprueba su propia vergüenza o dolor, no es probable que preste una atención seria a la angustia interna de los clientes. En situaciones como ésta, es más útil centrarse en las sensaciones corporales del cliente y luego en su afecto, que en los detalles de su historia.

Una indagación dirigida a que el cliente descubra sus sensaciones corporales debe estar basada en una seguridad terapéutica sutil que se dirija al nivel físico y emocional. A menudo, es adecuado animar al cliente a entrar y salir de su experiencia emocional intensa con un proceso terapéutico de apoyo, para que experimente un contacto emocional intenso durante un breve período de tiempo, y luego salga de nuevo con una sensación de confianza en que puede manejar la intensidad de sus 
emociones (Erskine y Mauriz-Etxabe, 2016). Se acompaña al cliente a manejar la intensidad de sus recuerdos llenos de afecto, y al mismo tiempo, a recuperar la información sensorial que existía en su cuerpo como resultado de un trauma o negligencias acumulativas. En este sentido, Erskine (2015) justifica la responsabilidad del terapeuta de centrarse en los procesos corporales, las retroflexiones, las reacciones fisiológicas de supervivencia y las estrategias de afrontamiento en la primera infancia (parálisis, agitación, retraimiento relacional).

Algunos clientes confían en su capacidad cognitiva para manejar su experiencia interior, están llenos de ideas o grandes planes como una desviación de sus memorias llenas de afecto. Estos clientes requieren mucha paciencia y una cuidadosa atención a cómo estas historias pueden ser metáforas para expresar el desánimo, la vergüenza o la soledad no expresadas. En resumen, la decodificación de las experiencias internas de nuestros clientes se logra a través de la indagación de la experiencia fenomenológica del cliente y de la formación de una narrativa histórica, lo que le ofrece la oportunidad de experimentarse así mismo de una manera nueva.

\section{Limitaciones de la Indagación}

A lo largo de la historia de la psicoterapia hay varios autores que afirman la importancia de la indagación en el proceso terapéutico. En general, están de acuerdo en que el vínculo, la confianza y las posibilidades de que el cliente pueda mejorar su calidad de vida se fomentan durante la misma, si esta se hace con respeto y sintonía con el afecto y el ritmo del cliente. Me pregunto si una de las limitaciones de la indagación se expresa en el siguiente poema:

No voy a la terapia para saber si soy raro.

Voy y encuentro una respuesta cada semana.

Y cuando hablo de terapia, sé lo que la gente piensa.

Que te vuelves egoísta y te enamoras de tu psicólogo.

Pero oh cómo amé a todos los demás cuando finalmente

Logré hablar tanto de mí mismo. (Van der Kolk, 2015, p. 229)

En la tabla 1, se presentan respuestas en la delimitación de obstáculos o barreras por parte del terapeuta que dificultan la indagación, impidiendo la escucha activa y el desarrollo del vínculo. Sin embargo, las respuestas 9 y 10 pueden ser nutritivas, ya que el terapeuta puede simpatizar, confirmar, consolar..., como forma de responder a las necesidades relacionales tanto arcaicas transferencialmente proyectadas sobre él/ella, como actuales. Y también hacer uso de la técnica de la indagación interpretativa o uso de la inferencia para ayudar a construir la narrativa del cliente. 
Tabla 1. Respuestas obstaculizadoras del Proceso de Indagación (adaptado de Gordon, 1970; citado por García, 2018, p. 24)

\begin{tabular}{ll}
\hline Respuestas & \\
\hline $\mathbf{1}$ & Ordenar, dirigir, o encargar. \\
$\mathbf{2}$ & Alertar o amenazar \\
$\mathbf{4}$ & Dar consejo, realizar sugerencias, o sugerir soluciones. \\
$\mathbf{5}$ & Persuadir con lógica, discusión, o enseñanza \\
$\mathbf{6}$ & Moralizar, sermonear, o decir a los pacientes lo que «deberian» hacer. \\
7 & Estar en desacuerdo, juzgar, criticar, o culpabilizar. \\
$\mathbf{8}$ & Estar de acuerdo, aprobar, o rogar. \\
$\mathbf{9}$ & Culpabilizar, ridiculizar, o etiquetar. \\
$\mathbf{1 0}$ & Interpretar o analizar. \\
$\mathbf{1 1}$ & Reafirmar, simpatizar, o consolar. \\
$\mathbf{1 2}$ & Cuestionar o poner a prueba. \\
\hline
\end{tabular}

Cuando la indagación terapéutica se hace en un tono de voz áspero o en un estilo de confrontación agresivo, las preguntas pueden ser recibidas por el oyente como crítica y avergonzante. Al ocurrir este hecho, la persona le da a exploración del psicoterapeuta un significado diferente al deseado. Por ejemplo, el cliente puede sentir vergüenza e interpretar la pregunta en el sentido de "no sabes”; “estás equivocado”; “deberías haberlo entendido tú mismo”. En muchas situaciones el cliente se siente avergonzado e incapaz de responder. En otras situaciones, el cliente puede repetir las diversas formas de hacer frente a la interrupción de las relaciones que aprendió en la infancia, como dar una respuesta superficial, endurecer su cuerpo o enfadarse en silencio. En otros momentos, interpretar los hechos que nos narran o hacer señalamientos o preguntas que despiertan la desconfianza pueden invalidar el darse cuenta original. Es importante que el cliente sea capaz de expresar lo que piensa y siente en esos momentos.

La indagación relacional se hace necesaria cuando hay una interrupción de contacto entre el cliente y el psicoterapeuta. La indagación relacional se compone de preguntas respetuosas sobre cómo el cliente experimenta la actitud, el comportamiento o las reacciones emocionales del psicoterapeuta. Estas preguntas son sobre el tono de voz, la velocidad y las diversas implicaciones que el cliente imagina mientras escucha la indagación. Es importante que facilitemos la capacidad del cliente para hablar sobre cualquier impacto que tengamos en él. La investigación relacional es sobre “tú y yo... y cómo nos relacionamos entre nosotros”. Se trata de 
nuestra interacción interpersonal. Como psicoterapeutas tenemos que preguntarnos a menudo “¿Cuál es el efecto de mi afecto y comportamiento en mi cliente?” (Erskine, 1995). A menudo aspectos significativos de la psicoterapia de un cliente surgen de una indagación relacional. Esta forma de diálogo nos lleva a asumir la responsabilidad de hacernos cargo de las posibles rupturas en la relación terapéutica.

\section{Conclusiones}

A lo largo de este artículo he descrito algunos de los aspectos de la indagación terapéutica que han sido importantes en mi trabajo como psicoterapeuta de orientación gestáltica y en psicoterapia relacional integrativa.

La indagación, en el proceso terapéutico, es un proceso flexible que requiere un ritmo único con cada cliente. Es necesario hacer nuestra indagación respetando la historia particular del cliente, en lugar de someterlo a la imposición de teorías psicológicas. Este proceso debe adaptarse a las necesidades del cliente.

La indagación terapéutica abre, para el cliente, posibilidades de experimentación que no han sido contempladas antes; y a medida que avanza, la conciencia y la espontaneidad se amplían en el cliente. Parte de la tarea, es utilizarla para apoyar el proceso y la creación del vínculo terapéutico entre el cliente y el psicoterapeuta. De esta manera, se convierte en una forma de aprender y entender los procesos intrapsíquicos e interpersonales del cliente. Este aprendizaje permite al terapeuta investigar el presente, lo que está sucediendo en el aquí y ahora, así como lo que ha ocurrido en la infancia del cliente y cómo se enfrentó a las diversas rupturas y traumas relacionales.

Al indagar en las historias de vida de cada uno de nosotros, encontramos recuerdos del pasado, que contienen mucha información, información registrada en el cuerpo, en el plano emocional, en la memoria cognitiva y en la forma en que nos comportamos. Estos recuerdos se construyen sobre la base de experiencias vividas de manera significativa para el niño. Cuando estas experiencias, son repetitivas o forman parte de una experiencia impactante o traumática, generan en el niño conclusiones sobre sí mismo, sobre los demás y sobre la vida. Conclusiones como "nadie me escucha", "no les gusto" o "soy inútil” se convierten en decisiones como "no voy a hablar", "no voy a amar a nadie" o "no estoy involucrado en lo que estoy viviendo”. Saber esto, facilita la comprensión, la ternura y la compasión hacia el cliente.

Hasta cierto punto, "la indagación comienza con la suposición de que el terapeuta no sabe nada sobre la experiencia del cliente y por lo tanto debe esforzarse continuamente por comprender el significado subjetivo de su comportamiento y proceso intrapsíquico" (Erskine, 2015, p. 17). En el mismo sentido, el terapeuta debe estar interesado en descubrir el sentimiento, el pensamiento y la actuación de su cliente a través de preguntas que estimulen la reflexión y el contacto del cliente. Una actitud de respeto, interés e implicación, como principales valores para y por nuestro cliente, anima al cliente a que las experiencias y necesidades insatisfechas, 
que en su momento se ocultaron, vuelvan a tener una base para poder llevarlas, otra vez, a su conciencia. La estimulación de los procesos cognitivos negados en base a recuerdos, sueños e ilusiones, entre otros, hace que recuperen el valor que tuvieron en su día y que probablemente se olvidaron o se convirtieron en indiferentes.

A modo de cierre de este artículo, subrayamos que el trabajo de la indagación terapéutica desde la bondad, la honestidad, el interés por la persona, la afectividad y otros valores que ofrecen seguridad, validación y protección brindan al cliente la oportunidad de alcanzar un estado de bienestar emocional y psicológico.

\section{Referencias}

Buber, M. (1958). I and Thou [Yo y Tú]. Scribner's.

Erskine, R. G. (1995). A Gestalt therapy approach to shame and self-righteousness: Theory and methods [Un enfoque de la terapia Gestalt para la vergüenza y la justicia propia: teoría y métodos]. British Gestalt Journal, 4(2), 107-117.

Erskine, R. G. (2004). Necesidades Relacionales. Instituto de Psicoterapia Integrativa. https://www.integrativetherapy.com/es/articles.php?id=21

Erskine, R. G. (2013). Vulnerability, authenticity, and inter-subjective contact: Philosophical principles of integrative psychotherapy [Vulnerabilidad, autenticidad y contacto intersubjetivo: Principios filosóficos de la psichoterapia integrative]. International Journal of Integrative Psychotherapy, 4(2), 1-9. http://www. integrative-journal.com/index.php/ijip/article/view/78

Erskine, R. G. (2015). Relational patterns, therapeutic presence: Concepts and practice of integrative psychotherapy [Patrones relacionales, presencia terapéutica: Práctica y conceptos de la psicoterapia integrativa]. Karnac Books.

Erskine, R. G. (2021). A healing relationship: Commentary of therapeutic dialogues [Una relación sanadora: Comentario de diálogos terapéuticos] [material no publicado]. Phoenix Publishing House.

Erskine, R. G. y Mauriz-Etxabe, A. (2016). Inference, re-experiencing, and regression: psychotherapy of Childego states [Inferencia, re-experimentación y regression: Psicoterapia de los estados de ego del niño]. En R. G. Erskine (Ed.), Transactional Analysis in Contemporary Psychotherapy (pp. 139- 159). Karnac Books.

Erskine, R. G., Moursund, J. P. y Trautmann, R. L. (2012). Más allá de la empatía (N. Mestre, Trad.). Descleé de Brouwer. (Trabajo original publicado en 1999)

Fagan, J. y Shepherd, I. L. (1970). Gestalt therapy now: Theory, techniques, applications [Terapia Gestalt ahora: teoría, técnicas y aplicaciones]. Science \& Behavior Books.

García, A. (Anfitrión). (2018-presente). Los 12 obstáculos de Thomas Gordon [Podcast]. https://alexgarcia.eu/ podcast/los-12-obstaculos-de-thomas-gordon/

Hycner, R. y Jacobs, L. (1995). The healing relationship in gestalt therapy [La relación curativa en terapia Gestalt]. The Gestalt Journal Press.

Macías, M. A., Orozco, C. M., Amarís, M. V. y Zambrano, J. (2013). Estrategias de afrontamiento individual y familiar frente a situaciones de estrés psicológico. Psicología desde el Caribe, 30(1), 123-145.

Moursund, J. y Erskine, R. G. (2003). Integrative psychotherapy: The art and science of relationship [Psicoterapia integrative: El arte y la ciencia de la relación]. Brooks/Cole.

Peñarrubia, F. (1998). Terapia Gestalt: La vía del vacío fértil. Alianza Editorial.

Pérez Porto, J. y Gardey, A. (2019). Indagar. En Definición.de. Recuperado el 22 de agosto de 2020. https:// definicion.de/indagar/\#: :text=Indagar $\% 20$ es $\% 20$ un $\% 20$ verbo $\% 20$ que,una $\% 20$ investigaci $\% \mathrm{C} 3 \% \mathrm{~B} 3 \mathrm{n} \% 20$ o\%20mediante\%20preguntas.

Perls, F. (1944). Ego, hunger and aggression: A revision of Freud's theory and Method [Ego, hambre y agresión: una revisión de la teoría y el método de Freud]. The Knox Publishing Co.

Perls, F. (1969). Gestalt therapy Verbatim [Terapia de la Gestalt palabra por palabra]. Real People Press.

Perls, F. (1973). The Gestalt Approach and Eye Witness to Therapy [Enfoque de la Gestalt y testimonio ocular de la terapia]. Science and Behaviour Books.

Perls, F. y Baumgardner, P. (1975). Gifts from Lake Cowichan: Legacy from Fritz [Regalos del lago Cowichan: Legado de Fritz]. Science and Behavior Books. 
Perls, F., Hefferline, G. y Goodman, P. (1951). Gestalt therapy [Terapia Gestalt]. New York, 64(7), 19-313.

Polster, E. y Polster, M. (1973). Gestalt Therapy Integrated: Contours of Theory and Practice [Terapia Gestalt integrada: Contornos de la teoría y práctica]. Brunner/Mazel.

Rosenfeld, E. (1978). An oral history of Gestalt therapy: I. A conversation with Laura Perls [Una historial oral de la terapia Gestalt; I. Una conversación con Laura Perls]. Gestalt Journal, 1(1), 8-31.

Van der Kolk, B. A. (2015). El cuerpo lleva la cuenta: cerebro, mente y cuerpo en la superación del trauma (M. Foz, Trad.). Eleftheria. (Trabajo original publicado en 2014)

Yontef, G. (1995). Proceso y diálogo en psicoterapia Gestalt (E. Olivos, Trad.). Cuatro Vientos. (Trabajo original publicado en 1993)

Zinker, J. (1978). Creative Process in Gestalt Therapy [Proceso creativo en terapia Gestalt]. Knopf Doubleday. 